\title{
Otolaryngological Complications of Hypopharyngeal-Esophageal Multichannel Intraluminal Impedance pH monitoring.
}

\author{
Francois Bobin ${ }^{1}$, Sven Saussez ${ }^{1}$, and Jerome Lechien ${ }^{1}$ \\ ${ }^{1}$ Affiliation not available
}

July 13, 2020

\begin{abstract}
pH-study is a routine procedure in some European laryngology offices to diagnose laryngopharyngeal reflux (LPR). Probe movements may complicate the testing leading to digestive symptoms and nose pains during the removal of the probe.
\end{abstract}

\section{Introduction}

Hypopharyngeal multichannel intraluminal impedance pH monitoring (HEMII-pH) with nasopharyngeal catheter placement is the standard of care for the diagnosis of gastroesophageal reflux disease (GERD) or laryngopharyngeal reflux (LPR) $[1,2]$. The approach is safe but may be awkward for patients. In a previous study, $61 \%$ of patients reported that the test bothered them most of the time, although $69 \%$ said that they would repeat the test if needed [3]. The placement of the HEMII-pH probe is made in-office and may be realized by both gastroenterologist and otolaryngologist. To date, a little number of studies reported complications of the procedure, which consisted of pain, discomfort or nose bleeding.

The aim of this paper is to report two unusual complications of HEMII-pH.

\section{Case 1}

A 65-year-old patient was referred to the Reflux consultation of the Elsan Polyclinic (Poitiers) for a chronic history of belching, postnasal drip, dysphonia, globus and heartburn. The nasofiberoptic examination found posterior commissure hypertrophy and arytenoid erythema. The history of the patient included refractory gastroesophageal reflux disease (GERD), while the gastrointestinal (GI) endoscopy reported Hill 2 hiatal hernia and esophagitis (grade A). Regarding the laryngopharyngeal complaints and the previous resistance to proton pump inhibitors (PPIs), a HEMII-pH testing (Versaflex-Z®), Medtronic, Europe) was realized to confirm the diagnosis. The HEMII-pH was composed of 8 impedance segments and $2 \mathrm{pH}$ electrodes. The catheter model used was introduced transnasally and considered the esophageal length of patient (GI endoscopy/manometry). Six impedance segments were placed along the esophagus zones (Z1 to Z6) and they were centered at 19,17,11, 9, 7 and $5 \mathrm{~cm}$ above the lower esophagus sphincter (LES). Two additional impedance segments were placed 1 and $2 \mathrm{~cm}$ above the upper esophagus sphincter (UES) in the hypopharyngeal cavity. The two pH electrodes were placed $2 \mathrm{~cm}$ above LES and 1-2 $\mathrm{cm}$ below UES. The probe was fixed to an external electronic data recorder that monitors the esophageal $\mathrm{pH}$. The association between symptoms and reflux episodes was studied: patient recorded the time of meals and the occurrence of key symptoms (belching, globus and heartburn) through the HEMII-pH device. The patient came back 24-hour after the placement of HEMII-pH catheter to remove it. He reported the occurrence of dysphagia 2 hours after the probe placement. The removal of the probe was associated with nose pain and the otolaryngologist discovered a distal probe node (Figure 1). The HEMII-pH tracing analysis showed a correct functioning of the system during the first hour of the testing period before the occurrence of several belching episodes, which were reported by the patient through the device. At this time, some parasites appeared in the tracing, reflecting 
the node formation (Figure 2). The rest of the recording confirmed the nonacid LPR diagnosis through the recording of the proximal probes, which were not impacted by the node. The patient consent was obtained for the publication.

\section{Case 2}

A 15-year-old patient with severe asthma was addressed to the reflux clinic to exclude ear, nose and throat disorders that may worsen asthma. The symptoms of the patient consisted of chronic cough, throat clearing and globus sensation. The fiberoptic examination reported edema and erythema of the posterior commissure. According to the clinical findings, HEMII-pH was performed to exclude the LPR diagnosis. The placement of the HEMII-pH catheter (Versaflex-Zß, Medtronic, Europe) and the characteristics of the device were similar to those reported in the first case. The patient had belching, nausea and several vomiting episodes during the probe placement. During the 24-hour testing, the patient reported many heartburn and belching episodes, which were not reported in the initial clinical picture. The removal of the probe was associated with nose pain and the physician found a folded probe (above Z5 segment; Figure 3). Tracing revealed parasites in the distal impedance segment throughout the 24-hour testing period. According to the tracing, the probe was probably folded during the vomiting episodes (placement time) and the related retrograde esophageal contraction. The rest of the recording confirmed the nonacid LPR diagnosis. The patient consent was obtained.

\section{Discussion}

The most common complications/adverse effects of HEMII-pH consist of nose pain, runny nose, nose bleeding, throat pain/discomfort, chest pain, cough, headache and dysphagia [4]. Catheter displacement is known to be a potential problem that may impair the test accuracy [5]. The $\mathrm{pH}$ probe movements usually depend on the body position, bolus size and composition and talking [5]. During swallowing, the $\mathrm{pH}$ probe began by ascending $0.5-2.0 \mathrm{~cm}$ then returned to the baseline position. A study found that, for 48 swallows, the ascending oscillation was followed by a descending oscillation of up to $2.0 \mathrm{~cm}$ before returning to baseline; the $\mathrm{pH}$ probe movements could account for the variation in results and reproducibility of simultaneous esophageal pH-monitoring. In this paper, we reported two rare complications of HEMII-pH characterized by probe movements related to retrograde esophageal peristaltic events (belching and vomiting). Regarding the features of the first patient, hiatal hernia could be a favoring factor. To the best of our knowledge, this kind of complication was never reported. Otolaryngologist who performed in-office HEMII-pH testing has to be aware about the risk of folded probe or probe node in patients with vomiting events or belching. The removal of the probe has to be cautious in patients with a clinical suspicion of probe movements regarding the theoretical risk of nasal traumatism.

\section{Conclusion}

In this paper, we reported two very rare complications of HEMII-pH, consisting of probe movements and node in the esophagus. These complications may affect the tracing analysis if the HEMII-pH testing is realized for GERD. For LPR, the impact is lower because the LPR diagnosis is mainly based on the occurrence of hypopharyngeal reflux episodes.

Acknowledgments: C. Knuts (Native Speaker U.S.) for the proofreading of the paper.

Conflicts of interest : authors have no conflict of interest.

\section{Author contributions :}

Jerome R. Lechien wrote the paper, analyzed the case, conducted literature review and performed the research. F. Bobin \& S. Saussez analyzed the case and wrote the part: "case report".

\section{References:}

[1] Chen CL, Orr WC. Analysis of 24-hour esophageal pH monitoring: the effect of state of consciousness. Curr Gastroenterol Rep . 2008; 10(3):258-62. 
[2] Lechien JR, Akst LM, Hamdan AL, et al. Evaluation and Management of Laryngopharyngeal Reflux Disease: State of the Art Review. Otolaryngol Head Neck Surg. 2019; 160(5):762-782.

[3] Fass R, Hell R, Sampliner RE, et al. Effect of ambulatory 24-h esophageal pH monitoring on refluxprovoking activities. Dig Dis Sci 1999; 44: 2263-9.

[4] Wong W M, Bautista J, Dekel R et al. Feasibility and tolerability of transnasal/per-oral placement of the wireless $\mathrm{pH}$ capsule vs. traditional 24-h oesophageal $\mathrm{pH}$ monitoring-a randomized trial.Aliment Pharmacol Ther 2005; 21: $155-63$.

[5] Aksglaede K, Funch-Jensen P, Thommesen P. Intra-esophageal $\mathrm{pH}$ probe movement during eating and talking. A videoradiographic study. Acta Radiol 2003; 44: 131-5.

Figure 1: The probe node .

Figure 1 footnotes : -

Figure 2: The hypopharyngeal multichannel intraluminal impedance pH-monitoring tracing .

Figure 2 footnotes : Impedance is represented by a colorimetric scale from $800 \mathrm{ohms}$ (white color, low impedance) to $10,000 \mathrm{ohms}$ (blue color, high impedance). The grey color consists of a non-physiological impedance value $>10,000 \mathrm{ohms}$, which is a parasitic tracing. From 8:00 to 8:52, the tracing revealed gaseous reflux episodes related to belching. From 8:52, a grey area appeared at the Z4 impedance segment, which consists of the node development.

Figure 3: The Folded probe .

Figure 3 footnotes : -

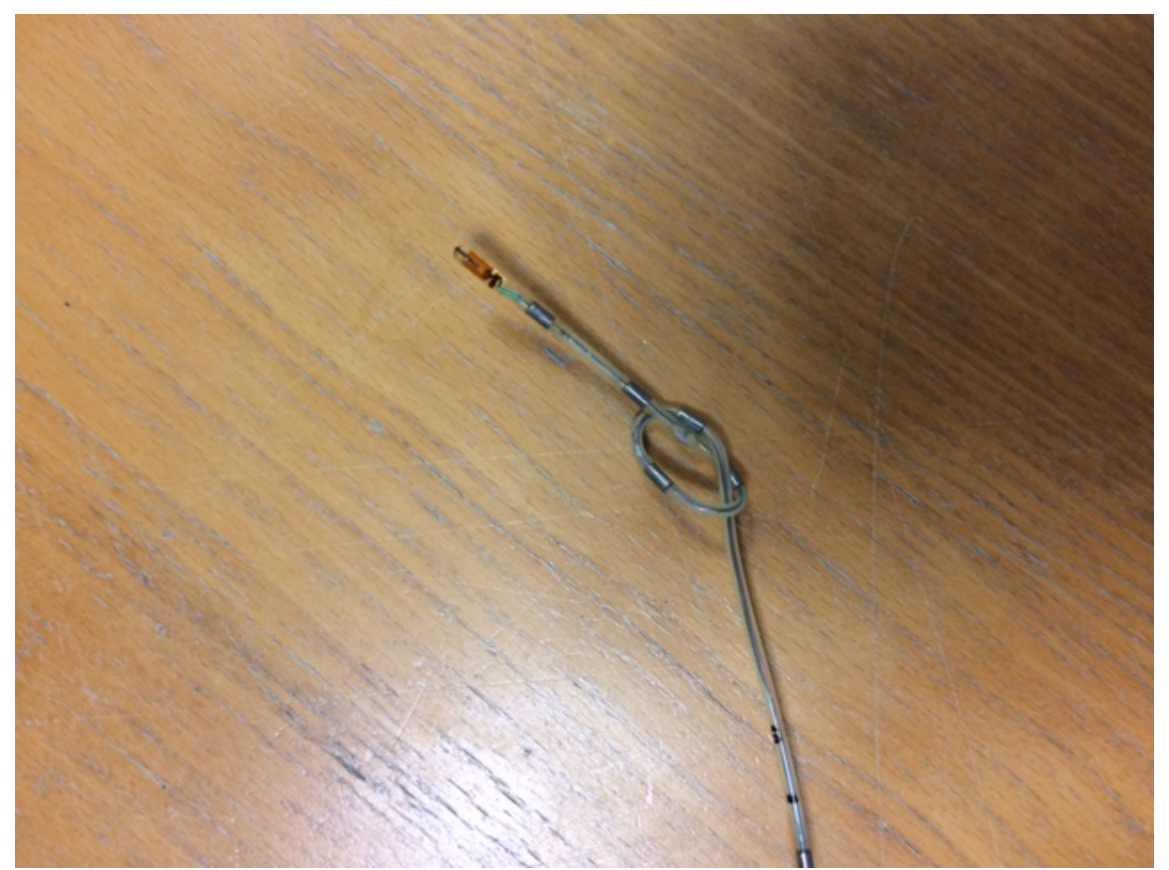



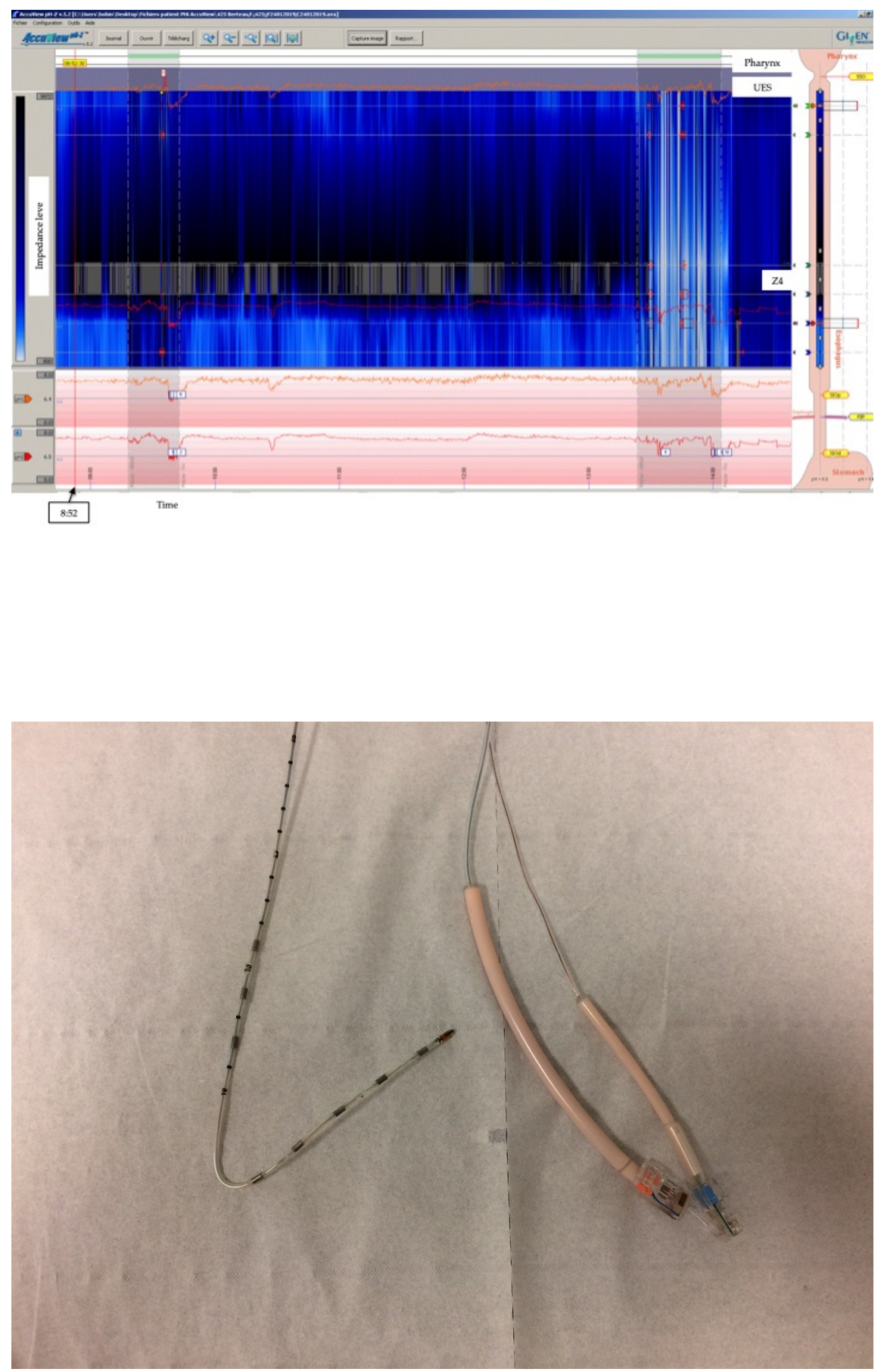